\title{
Le teologie comunicanti di Giuseppe Bonaviri, autore delle Novelle saracene
}

Maria G. Vitali-Volant

\section{OpenEdition}

Journals

Edizione digitale

URL: http://journals.openedition.org/cei/210

DOI: $10.4000 /$ cei. 210

ISSN: 2260-779X

Editore

UGA Éditions/Université Grenoble Alpes

\section{Edizione cartacea}

Data di pubblicazione: 15 juillet 2009

Paginazione: $211-220$

ISBN: 978-2-84310-145-8

ISSN: 1770-9571

Notizia bibliografica digitale

Maria G. Vitali-Volant, «Le teologie comunicanti di Giuseppe Bonaviri, autore delle Novelle saracene», Cahiers d'études italiennes [Online], 9 | 2009, online dal 15 janvier 2011, consultato il 26 mars 2021.

URL: http://journals.openedition.org/cei/210 ; DOI: https://doi.org/10.4000/cei.210 


\title{
LE TEOLOGIE COMUNICANTI DI GIUSEPPE BONAVIRI, AUTORE DELLE NOVELLE SARACENE
}

\author{
Maria G. Vitali-Volant \\ Université du Littoral - Côte d'Opale (Dunkerque)
}

Le Novelle saracene, scritte da Giuseppe Bonaviri nel 1980, cominciano con il racconto di Gesù e Giufá dove:

Maria nella sua bottega vendeva brocche, brocchette, bòmboli, vasi e vaselli che i carrettieri portavano in paesi stranieri [...].

Un giorno diventò incinta, aspettava un figlio, e la sua anima si perdeva per lumi di luna su cespi e rovi. Lasciò la bottega e si recò su un monte dove adorando Dio voleva partorire in solitudine. Il padre forse era Milùd [In lingua libica, significa Natale], arabo, figlio di Zacri [...].

"E come lo chiamo?" si disse. Fuori in tempesta era la notte.

Sentì una voce: "Chiamalo Gesù" (Bonaviri, 1995, p. I5).

Il bimbo della storia viene abbandonato e allevato da una pecora. Ormai grandicello, arriva nel villaggio dove incontra il fratello di Maria, lo scarparo Michele Gabriele che "spiegava [ai bambini] come nasce l'Ora, come muore l'Uomo, e dopo se ne va a camminare nel prato asfodelo, insegnava i movimenti dei mondi sparsi per il cielo" (ibidem, p. 20), uno scarparo cherubino, o divinità dalle scarpe alate, piuttosto, che porta il nome dello zio dell'autore, il calzolaio Michele emigrato in America. Non a caso quindi, quando i bambini saraceni vedono Gesù per la prima volta in compagnia di Michele Gabriele, cantano "Bambinello, balla, balla, / tutta l'aria è tutta tua; / dove metti il tuo pieduzzo / nasce menta e basilicò" (ibid., p. I7). Nelle Novelle saracene la gente parla molto: "Ciù, ciuciù, pipipì, ciùciùciù, rococò" e questo parlare, consigliare, intervenire, partecipare al e nel racconto testimonia della vitalità popolare; è l'omaggio dell'autore alla sua gente in contrapposizione al silenzio delle genti di Sciascia murate nell'omertà. Oltre al parlare c'è il canto: quando i poveri del paese sanno che Giufa-Gesù cambia le pietre in pane: "Turi Cacuòcciula (Carciofo), / 
e Ciccio Pisciacane, / Iano alliccapòsperi (leccafiammiferi) / e Puddo lu sgummàto (gobbo); / Peppi 'Mpalaporte (Impalaporte) / e Matteu l'ugghiulàru (venditore d'olio) / e Iano sucaspàrici (succhiasparagi) / e Angiuzzu 'u cicuraru (il cicoraio)" (ibid., p. 28) cantano la loro storia in questi termini:

Quando, Gesuzzo, nascemmo noi sventurati, / fu nostra la mala ventura. / Per tre giorni stette il sole nascosto / e per quattro giorni non uscì la luna. / Chi sventurato nasce / in quello stato muore; / chi ventura non ha, / non ne può avere. (Ibidem)

Così pregano, lo sollecitano per avere pane, quindi lo riconoscono come "adorabile" essere divino che interviene nella loro realtà e a loro beneficio. Un senso del religioso che esula dai misteri, che non conosce la paura degli idola e si allaccia ai bisogni materiali dell'esistenza. Quando, nella storia di Gesù, Maria infine ritrova suo figlio, le donne saracene cantano: "O ferita dolorosa / diventata ormai gioiosa! / O amor divino, o amore / dilettabile e amabile! / O Gesù nostro agnello bello!" (ibid., p. I8). Qui le donne sacralizzano l'amore della madre, un tema che sta alla base della religiosità bonaviriana e mediterranea ancestrale. Con un ritmo di favola familiare, di melopea e laude jacoponiana, comincia la storia dove si intrecciano tutte le mitologie, i racconti del sogno di avventure e di gloria e le teologie, dal mondo classico al Vangelo, passando per i testi sacri del mondo arabo ed ebraico. Ma la storia è una e sempre quella e comincia in un paese lontano lontano, ma così vicino all'autore perché si tratta di Minèo sui monti Erei, dove egli è nato nel 1924. Un villaggio di una Sicilia pietrosa, legata alla tradizione dell'ulivo, del carrubo e alle divinità ctonie. In questo paese, i contadini sono angariati dal loro signore e anche dagli altri signorotti del luogo, ci si mette anche il Papa e la lotta per la sopravvivenza si fa sempre più dura; per vincere la paura e sconfiggere la fame e il dolore, si raccontano storie che prendono origine dai testi tramandati oralmente da generazioni di uomini pii e sapienti dei ritmi della vita: i testi sacri e i poemi epici, uniche fonti di ispirazione dei poveri. Sul loro canovaccio si intessono i fili di seta del tappeto animato che il poeta siciliano s'incanta a ricreare pescando nella memoria dei racconti che sua madre gli faceva da piccolo. Nella nota al testo l'autore dice:

Con questi racconti mi pare ritrovare con la mia gente e con mia madre un preciso punto di incontro nel nostro profondo sentire.

Difatti il novellare è un perpetuo inseguimento del Desiderio, e nella mia terra rappresentava un sacrale e smarrente involucro, quasi una placenta, dentro cui attraverso il narrare orale di artigiani e contadini finivamo col ritrovarci. [...] la loro matrice siciliana si trova in una solarità mediterranea che vi è diffusa, nelle funambolerie delle ballate, e ballatelle, nelle laude irridenti, o drammatizzate, in vibrazioni mitologiche. (Ibidem, Nota, p. 193) 
La gran parte di queste novelline fanno parte d'un patrimonio etnografico euroasiatico, per non dire universale, ma indubbiamente hanno subito varianti notevoli, cadenze, recitativi tipicamente siciliani. Come, d'altronde, si può notare nella prima parte di questo libretto dove si trovano moltissime stratificazioni storico-etniche, e magiche, di un epos cavalleresco del contado siciliano. Così, madruzza, troviamo il candido [nel senso volterriano, diciamo noi] Giufa accanto ad Orlando, che era simbolo di peregrinante forza, e insieme a Gesù reso nella sua originaria figura di saraceno giramondo: come se il nostro villano, fermo per secolare destino tra borgo e terre vicine, si fosse voluto creare una Divina Trinità da eterodossi personaggi pronti, sempre nel loro girovagare, a fare storia o con la spada durlindana, o col buffo candore, o con un apparente gioco di miracoli fiorenti in modo perenne dalla mente del villano-Gesù. Al punto che re Federico II di Svevia [...] perseguita questo Gesù saraceno, sottoponendolo in ultimo a tortura, con i falchi, assieme a Giufa e a Orlando [infine sottomessa la Sicilia nelle sue tre anime popolari, ancora noi]. Insomma si rovescia la usuale posizione cristiana - e del pensiero cristologico - per rifarsi ad un dramma pagano, o forse pre-cristiano, quando s'ebbero a scontrare due culture, due egemonie, due opposte mitografie. Ogni cosa subisce un rovesciamento [un altro, fra i mille giochi del rovescio della nostra letteratura, sempre noi], in questo caso, persino il tempo e lo spazio e il modo d'intendere il Divino. (Ibid.)

Le novelle di questo medico-narratore-poeta si ispirano infatti alla tradizione popolare di tutto il Mediterraneo e l'itinerario letterario di Bonaviri si snoda attraverso una affabulazione trasversale in cui le influenze arabe, giudaiche e cristiane disegnano strani percorsi narrativi e toccano al sacro come esperienza comune, come vissuto di popoli pre-religiosi. La parola-suono di Bonaviri è il canto del pastore Gesù, del calzolaio Giufa o la melopea di Michele Gabriele. Un mondo che Renan farebbe risalire al Kitab-et-Aghani (Livre des Chansons), immagine della vita araba libera prima di Maometto, in cui si ritrovano le scene e i personaggi delle Leggende patriarcali (Renan, pp. XVI-XVII) giudaiche. La narrazione fiabesca di Bonaviri tesse legami e inventa favolose teologie comunicanti di un popolo di umili che si identifica nelle sue divinità, "creature naturali, esseri corporei, ragionevoli ma diversi” (Veyne, p. 425), che coabitano con gli uomini nello stesso mondo. Bonaviri, alla ricerca dell'identità e delle origini, viaggia indietro nel tempo per ricostituire il poema dell'epifania della sua terra. Mosso dal mito etico ed estetico di chi crede nella bellezza e nella verità che si cela nelle usanze e detti e canti e favole della povera gente illetterata, Bonaviri si ispira, per le sue fonti siciliane, alle opere di un altro celebre medico-studioso: Giuseppe Pitré. Ma se quest'ultimo inserisce la ricerca insulare nella corrente degli studi europei, Bonaviri estende la ricerca al di là dell'Europa e sconfina nelle terre dei nemici di sempre, gli infedeli invasori, adoratori del dio Macone, divinità antagonista che troviamo nei Poemi cavallereschi dell'Aretino, nel Morgante del 
Pulci, nella Gerusalemme liberata del Tasso (Tasso, II, 2, v. I), nell'Orlando innamorato del Boiardo (Boiardo, passim) e poi nel rifacimento del Berni che, come Bonaviri, architettava un "mondo capovolto" in cui il più debole nel "secol d'oro" potesse vivere nel "celeste / stato innocente primo di natura" (Berni, Capitolo primo della peste). Il genio poetico di Bonaviri avvicina ciò che la storia ha diviso e divide ancora, fa convivere nel microcosmo di Minèo le divinità e gli eroi dei popoli che si affacciano sul mare comune, così come utilizza i generi diversi della tradizione popolare: l'apologo sempre legato alle circostanze pratiche del vivere, la leggenda spesso legata ai luoghi, l'agiografia, il racconto moralistico di stampo volterriano e la fiaba che mette in primo piano i capovolgimenti dello spirito cristiano attuati dal costume popolare. Nel suo libro Sulla fiaba, Calvino nota che nelle opere Canti popolari della Contea di Modica (1876) e La parità e le storie morali dei nostri villani (I884) del siciliano Serafino Guastella, nobiluomo letterato della fine dell'Ottocento "che scrive solo di costumi contadini" (Calvino, pp. 65-8I; qui p. 66) troviamo un cristianesimo rovesciato con incarnazioni inaspettate: san Paolo è un barbiere erudito e profeta, san Gerlando fa il ladro di mestiere, san Giuseppe va a rubare i fichi con Gesù bambino (ibid., p. 74). Per Bonaviri san Pietro suona il violino, san Giovanni la trombetta e Gesù suona la bombarda in una banda contadina che annuncia la buona novella a Qàlat-Minaw (Zangrilli, p. 4I), Minèo in arabo, dove si vive la "malannata" (Bonaviri, I995, p. 5I). Un ennesimo segno che, nel mondo rurale universale, il leggendario delle divinità si capovolge e addomestica, in una rappresentazione delle necessità umane: all'ascetismo e al martirio si sostituiscono le astuzie per la sopravvivenza in un mondo precario in cui le disgrazie sono già la sorte collettiva. Le scelte letterarie di Bonaviri, la sua scrittura narrativa, sigillano il suo stile d'autore, la sua autonomia rispetto alla prosa erudita, e fissano l'originalità dello stile eclettico, che predilige i toni della lirica, le sfumature ironiche e la parodia, lo stile delle fiabe metafisiche e cosmogoniche, o della poesia burlesca del secolo dei Lumi, innovatrice e sincretica, e anche della prosa scientifica del Settecento siciliano: i trattati di zoologia e di mineralogia di Gioeni, per esempio. Il tutto, sempre ispirandosi a una letteratura fra "il primitivo e l'arcadico, dove il valore poetico viene dal senso delicatamente cosmico con il quale rappresenta il piccolo mondo di Minèo" (Bonaviri, 1954, p. I2) come diceva Vittorini nella presentazione del Sarto della stradalunga del 1954 (primo romanzo edito di Bonaviri). Eppure in questo paese sperduto si trova la "pietra della poesia": una roccia animata intorno alla quale si riunivano i poeti contadini e artigiani da ogni parte della Sicilia, per gareggiare recitando versi. "C'è da pensare - dice Bonaviri - 
che questa pietra riportasse a una religione non solare, celeste, bensì sotterranea, fondata sulla convinzione che le divinità risiedessero nella terra" (Tiberia, p. 5). Intorno a questa pietra sacra si è formato il "cerchio delle fate" della poesia di Bonaviri, poesia-verbale, suoni che preesistono alle cose come le divinità ctonie dell'episodio la Luna di Gesù dove "Dio è una pietra-calamita: attrae e allontana le cose, non ha sensi, né respiro, né gioia” (Bonaviri, 1995, p. 49) o dell'altra fiaba cosmica La divina foresta del I969, in cui parlano gli animali o le metamorfosi dell'umano nel divino della natura, e il ragno-femmina Isinera, si:

[...] presentava come un essere ctonio che in quanto tale, non credeva né nel numero, né nella causa, né nell'acqua, né nel fuoco [...] ma nelle emanazioni oscure e nelle tenebre che si trovano sempre sotto terra [...]. Nel suo regno non si incontrava nessuno, salvo le tenebre che nella loro innocenza rappresentano il prologo e la fine di tutte le cose. (Bonaviri, 1969, p. 95)

\section{Nelle Novelle, Bonaviri celebra l'epifania degli:}

[...] Dii: [...] piccoli, piccolissimi, tutt'aggrondati e travagliati [...].

Quelli là, gli Dii, ruotarono, fecero inverno, primavera, ma erano tutti in paura, gliela aveva tramandata il Padre. [...] Non potevano controllare la infidezza del loro gonfiare, sprofondavano nel mare delle cose. Fili lunghissimi li univano per grani di forte paura, erano tutta una paura; gridavano per spavento, senza intelletto o virtù divina. Si spappolavano in scintille, lasciavano nell'aria zampe, corsaletti, occhi, ali, alucce, polvere in gran splendenza. Ma morivano; in primis il Dio Padre, dopo il Dio Figlio, poi quelle Dee Femmine, e le femmine delle femmine; diventavano semplicetta fiamma in mezzo alle stelle. Appena uno moriva, ne nasceva un altro, differente, s'aggomitolava in fumo, era sussurro, spiga, gli dii-femmine si inanellavano, erano tanti spiritelli piangenti. Così diceva Gesù. Così sia. (Bonaviri, I995, p. 50)

Questo Gesù di Bonaviri è saraceno, e si sdoppia nel magico Giufa protagonista del folklore narrativo arabo-islamico col nome di Djuha che diventa il Giochà ebraico, l'innocente che cammina sulle acque (Di Leo, I996, nota 20, p. I4I). La cultura saracena ha lasciato in Sicilia tracce di una civiltà prestigiosa, alla corte palermitana di Federico II di Svevia, il tiranno delle Novelle, venivano accolti e onorati grandi scienziati e artisti saraceni, sicché la tradizione cristiana si mescolò con quella musulmana. Bonaviri però ritrova questa ecclesia federiciana nel "popolo dei vinti" dove non ci sono scienziati ma maghi come Giufa o poveri cristi come Gesù. Il Giufa di Bonaviri è un personaggio contradittorio, una divinità che si manifesta in astuzie, furberie, bricconate insensate, miracoli, oracoli e profezie di saggezza sopranaturale; dorme trecento anni in fondo a un pozzo e, con le pietre che gli sono cadute addosso "criptio, fermezio, tellurio, iridio, nubilio in tristezza...” (Bonaviri, 1995, p. 32) - il linguaggio-suono 
(Bonaviri, I969, p. 8) di Bonaviri diventa gergo alchemico - crea la luna che in "dolce lamento cammina sotto" (Bonaviri, I995, p. 46) il pianetacosmo Minèo. Il percorso narrativo di Bonaviri ritorna sempre al punto di partenza. Nella novella Il fratello di Gesù, Egli, stanco di camminare per il mondo sempre in pena e in guerra in compagnia di Gioacchino e di san Francesco, resuscita tre amici d'infanzia saraceni che re Vittorio (all'improvviso ci troviamo nel Risorgimento) aveva fatto condannare a morte e, per salvarsi dai gendarmi, si trasforma nel ferro rovente della porta di Bacco di Minèo. Insomma, la pratica religiosa di Bonaviri si concentra nel tempio delle origini, nel materno recinto sacro, in una religione panica, nei culti della terra che interpretano la rinascita ma anche la morte. E la malinconia e la morte sono i numi tutelari di tutta l'impresa narrativa di Bonaviri. La morte bonaviriana mette in contatto con un soprammondo indefinibile, imprecisabile di vaganti energie, è un'immissione del divino sia nelle religioni solari che in quelle sotterranee. Morte concreta e corporale del figlio del Sarto della Stradalunga e morte del mondo per opera di Gesù scontento di sé e degli uomini; morte sacra, rito sacrificale estremo per Giufa, Gesù e Orlando ${ }^{1}$, simboli dello spirito della Sicilia popolare che viene frainteso, sradicato e annientato dalla violenza dei dominatori di sempre.

Le Novelle saracene rappresentano, in chiave altamente poetica ma in una dimensione profondamente umana, la sintesi di una civiltà composita e di una storia fuori del tempo. Anche se si situano in un luogo - la Sicilia - appartengono ai "[...] racconti di meraviglie magiche, che non ammettono d'essere situate nel tempo" (Calvino, p. IIo). Non sono fiabe per ragazzi, ma suggeriscono la lettura evangelica dei fanciulli che possiedono le chiavi del Regno. Il Logos bonaviriano delle Novelle è frutto di un impasto linguistico di una creatività barocca nel senso datole da Genette (Genette, p. 89) di amplificazione del racconto in forma di poesia, di lamento, orazione funebre, laude, filastrocca, che accentuano la drammatizzazione nel tessuto narrativo, come nel racconto della Resurrezione di Giufá dove sua madre Maria Maddalena e anche la "signa Maria madre di Gesù" gridano a Giufa, capo dei villani impegnati in una battaglia tellurica contro le armate di "Recàne", alias, il solito Federico di Svevia: "O figlio del mio ventre, / meraviglia di nuova gente, / di Sicilia e Castiglia Re, / uccidi Federico, fallo defunto subito!" (Bonaviri, I995, p. 35), oppure

I. La morte di Gesù, in Bonaviri, 1995, pp. 62-70. Orlando, Gesù e Giufa vengono catturati da re Federico e crocefissi a tre alberi di olivo, sottosposti alla tortura dei falchi come Prometeo; quando muoiono, i poveri cantano "O Nostra Trinità, Gesù, Giufa, Orlando, ve ne andate nella fossa oscura. Il mondo non ci sarà più". 
quando Giufa si fa apostolo di Gesù per imparare di nuovo a fare miracoli prima di diventare re di Sicilia al posto dello Svevo: "Nessuno ebbe più fame, / tutti furono in sogni, / schietti (non sposati) e maritati! / Ognun si lusingò, / la fame se ne andò, / tutti in bellezza, / senza vecchiezza" (ibid., p. 39). Gesù è simmetrico di Giufâ, quest'ultimo nato "con difetto di cervello, non capiva bene, pensava solo a mangiare pane spalmato con olio e pepe" (ibid., p. I9), una sorta di semidio greco o romano, un Ercole - jiin tutto forza debordante e insensata, l'incarnazione della natura, che è insieme benevola e malefica, dea delle stagioni e dei raccolti ma anche portatrice di carestie. Il mitema (Calvino, p. III) Giufá e Gesù rimanda al mito di Cerere e Proserpina, due facce della stessa divinità, in bilico fra umano e sovrumano. Continua Bonaviri: "[Gesù] non aveva la potenza di Giufa. Diceva per esempio al passero: 'Diventa merlo'. Ma quello in lode di dio Macone restava passero. Diceva a una pietra 'fatti pane', la pietra in piangente cuore s'alzava ma non si trasformava in pane" (Bonaviri, I995, p. 27), la potenza di Giufa è contrapposta alla dolcezza di Gesù, alla sua soave malinconia, che si manifesta nell'opera di Bonaviri Gesù e il bambinello del 1987, in cui ritorna il tema narrativo dell'erranza del Nazareno insieme agli apostoli, ma in un contesto urbano, dove, a contatto del dolore umano in un ospedale pediatrico, egli non potrà intervenire come nelle Novelle saracene sotto forma di Giufa o grazie agli incantesimi di Michele Gabriele, e il solo miracolo di questo dio invecchiato, pieno di acciacchi, seguito da apostoli anche loro incanutiti e fragili, piangenti e deboli, sarà la contentezza, lo stato di grazia degli spiriti innocenti e l'attesa, come nel Giardino degli Ulivi, dell'intervento del Padre.

I punti storici di riferimento della favola di Gesù e di Giufá sono tre: Minèo; re Federico perché nei canti popolari è il sovrano più celebrato in Sicilia; Orlando perché è l'eroe dei cantastorie siciliani. Gesù, Giufa e Orlando costituiscono dunque la Trinità, crocifissa su tre alberi di ulivo. Qui il mito riprende la crudeltà del reale e trova applicazione, in maniera evidente, quel realismo delle immagini che si lega alla tradizione letteraria ottocentesca in Sicilia, dove la favola diventava fiaba, cioè fantasia. Bonaviri cita spesso Luigi Capuana, autore di fiabe e anche lui, nato a Minèo nel I834. Per Bonaviri tutto diventa favola nel senso aristotelico di "imitazione dell'azione" o trasformazione della natura, onde il verosimile si assimila al vero come voleva il Tasso, nel caso di Bonaviri un verosimile ingenuo e primitivo. Calvino, in una lettera a Bonaviri del 1969, a proposito della Divina foresta, si felicita con l'autore perché con lui “[...] la letteratura italiana ritrova quella che era la sua vocazione specifica nei 
suoi primi secoli: letteratura come 'filosofia naturale"' (Uno scrittore come Bonaviri, p. 8).

Nelle Novelle, nonostante le apparenze, non c'è ombra di volontà blasfematoria ma una profonda religiosità che si esterna nella compassione, nel senso di "comunanza di dolore" ${ }^{2}$, una pietas contadina rispettosa della memoria dei padri assunta da Bonaviri nella commemorazione del padre, punto di irradiazione di tutta la sua opera, don Nané: il "sarto della stradalunga" di Minèo, il poeta di Arcano, raccolta di versi rispettosamente e amorosamente trascritti dal figlio nel I975. Una religiosità filiale che accomuna la madre mediterranea, dea della vita e della morte, signora della parola narrata, alla figura del padre, divinità sovrana, "più grande che in natura" (Piaget, p. 297, p. 317), ma sempre compassionevole.

Per l'autore, il raccontare instaura uno spazio di resistenza, di riscatto, costruisce un lessico di opposizione alla cultura dominante. Bonaviri si ritrova spesso in una dimensione militante, che gli deriva anche dalle sue scelte politiche: nel 1944, aderisce al Partito Comunista. Scelta importante perché "dissacrante, fu come un risvolto aggressivo-pensoso verso la realtà che il fascismo, e il tardo Ottocento, ci lasciava" (Zangrilli, p. 90). L'impegno di Bonaviri si manifesta in una visione della Sicilia ricca di religiosità e di comprensione umana, arcaica, dolente e saggia. In questo universo, che affonda le sue radici nel mondo contadino, i drammi si "consumano" lentamente e le lotte si vivono all'interno della comunità rurale, familiare. Gli uomini di Bonaviri non sono mai soli e non perdono la speranza. Questa coralità trasversale, che abbraccia tutta la sua opera, prende le voci e gli idiomi di tutti coloro che esprimono il desiderio della parola come comunicazione e messaggio salvifico. Il suo è un canto orfico dalle cadenze dialettali siciliane, arabizzanti o giudaiche, espresso in un italiano-sabir, poetico e musicale, quasi una lingua rabelesiana dalle risonanze mediterranee. In questo narrare ricco di metafore, asindeti e ossimori, si evocano per contrasto le immagini di un paesaggio arso dal sole, di rari corsi d'acqua, di valli scoscese dove prendono corpo, e parola, piante, metalli, animali in forma di allucinazioni fiabesche, evocazioni animistiche, e anche odori: di spezie, di fiori, del cibo, soprattutto del pane che evoca il nutrimento celeste ma che è anche il solo "dolce amico" dei poveri. Questo lessico "sensista" (nel senso dell'esperienza sensibile del sensismo settecentesco) e sensuale è di una estrema modernità e testimonia della volontà dell'autore di avanzare nella ricerca linguistica su strade mai

2. Dal latino cristiano compassio, derivato di patire. 
percorse prima: un'avventura espressionista che disloca piani e superfici narrative, con l'intenzione di perturbare la tradizione dello scrivere ma non il sacro tessuto della memoria. Siamo arrivati alla fine con un "Basta", parola di sospensione del racconto, ampiamente utilizzata da Bonaviri e dai cantastorie quando il tempo stringe.

\section{Bibliografia}

Aretino P., Poemi cavallereschi, a cura di D. Romei, in Edizione nazionale delle opere di Pietro Aretino, Roma, Salerno, I995.

Boiardo M.M., Orlando innamorato, a cura di R. Bruscagli, 2 voll., Torino, Einaudi, I995.

Berni F., Orlando innamorato di Matteo Maria Boiardo rifatto da Francesco Berni, 2 voll., Torino, M. Guigoni, I858.

Bonaviri G., Gesù e il bambinello, Roma, Cometa, 1987.

—, Il sarto della Stradalunga, introduzione di E. Vittorini, Torino, Einaudi, "I Gettoni", I954.

—, La divina foresta, introduzione di G. Manganelli, Milano, Rizzoli, 1969.

—, Novelle saracene, introduzione di G. Gramigna, Milano, Mondadori, 1995 (prima edizione Milano, Rizzoli, I980; tr. fr. Contes sarrasins, trad. par J. Bloncourt-Herselin, Paris, Denoël, 1985).

Calvino I., Sulla fiaba, Torino, Einaudi, 1988.

Cohen Sarano M., Storie di Giochà, Firenze, Sansoni, I990.

D’Angiò G. (duca Giuseppe), Saggio di Lithologia Vesuviana, Napoli, Stamperia Vesuviana, I790.

-, Descrizione di una nuova famiglia di testacee, trovate nel litorale di Catania, Napoli, I783.

Di Leo M., Le storie di Giufá, Palermo, Flaccovio, 1996.

Genette G., Figures II, Paris, Seuil, 1969.

Piaget J., La représentation du monde chez l'enfant, Paris, Alcan, 1938.

PItré G., Biblioteca delle tradizioni popolari siciliane, Palermo, Pedone, I87I-I9I3.

-, Fiabe, novelle e racconti popolari siciliani, raccolti e illustrati da Giuseppe Pitré, ristampa anastatica Bologna, Forni, 1969.

Pulci L., Morgante e Lettere, a cura di D. De Robertis, Firenze, Sansoni, I984. 
Renan E., Légendes patriarcales des Juifs et des Arabes, cours professé au Collège de France I888-I889, introduction par L. Rétat, Paris, Hermann, I989.

Tasso T., La Gerusalemme liberata, a cura di A. Carini, Milano, Feltrinelli, I96I.

Tiberia R., Alla ricerca di una poesia biologica, intervista con Giuseppe Bonaviri, <www.italialibri.net/interviste/0502-I>.

Uno scrittore come Bonaviri. Iconografia, biografia di Giuseppe Bonaviri con 2 lettere di Elio Vittorini e Italo Calvino, un testo di Giorgio Manganelli e 3 poesie inedite di Giuseppe Bonaviri, Roma, Cometa, 1995.

Veyne P., L'Empire greco-romain, Paris, Seuil, 2005.

Zangrilli F., Conversazione con Giuseppe Bonaviri, Ravenna, Longo, 1998. 